\title{
Raw Dataset for Interim Analysis
}

National Cancer Institute

\section{Source}

National Cancer Institute. Raw Dataset for Interim Analysis. NCI Thesaurus. Code

C115495.

The collection of unprocessed data records used for an interim analysis of results during a clinical trial. 